\title{
LipidBuilder: A Framework to Build Realistic Models for Biological Membranes
}

\author{
Christophe Bovigny ${ }^{1,2,, \#}$, Giorgio Tamò ${ }^{1,2 \#}$, Thomas Lemmin ${ }^{1, \$, \#}$, Nicolas Maïno ${ }^{1}$, and Matteo
} Dal Peraro ${ }^{1,2^{*}}$

${ }^{1}$ Institute of Bioengineering, School of Life Sciences, Ecole Polytechnique Fédérale de Lausanne (EPFL),

${ }^{2}$ Swiss Institute of Bioinformatics (SIB), CH-1015 Lausanne, Switzerland

${ }^{*}$ Corresponding author: Matteo Dal Peraro

EPFL SV IBI LBM, AAB 010

Station 19, CH-1015 Lausanne

Email: matteo.dalperaro@epfl.ch

Phone: +41216931861

\# These authors equally contributed to this manuscript.

${ }^{\S}$ Current address: Molecular Modeling Group, SIB Swiss Institute of Bioinformatics, Quartier Sorge, Batiment Genopode, CH-1015 Lausanne, Switzerland

${ }^{\$}$ Current address: Department of Pharmaceutical Chemistry, University of California - San Francisco -UCSF, San Francisco, California 


\section{Supporting Figures and Tables}

Table S1. List of MD simulations for pure POPC, POPE, DOPC and DPPC lipids (see also Table 2 and Figure 3), and for the three realistic systems.

\begin{tabular}{lccc}
\hline Simulations & $\begin{array}{c}\text { Time of Simulations } \\
{[\mathbf{n s}]}\end{array}$ & $\begin{array}{c}\text { Numbers of } \\
\text { Lipids }\end{array}$ & Counter ions \\
\hline POPC & 100 & 81 & - \\
\hline POPE & 100 & 75 & - \\
DOPC & 100 & 66 & - \\
DPPC & 100 & 79 & - \\
Phage PRD1 & 62 & 464 & $\mathrm{NaCl}$ \\
Salmonella enterica & 63 & 476 & $\mathrm{NaCl}$ \\
\hline Chlorella spp. & 73 & 374 & $\mathrm{NaCl}$ \\
\hline
\end{tabular}


Table S2. Composition and properties of Salmonella enterica membrane (see also Figure 4).

\begin{tabular}{llccc} 
& \multicolumn{1}{c}{ lipid nomenclature } & concentration (\%) & area/lipid & std error \\
\hline PE 36:2 & $18: 1(Z 11) / 18: 1(Z 11)$ & 4.62 & 60.11 & 3.14 \\
\hline PE 30:1 $14: 0 / 16: 1(Z 9)$ & 9.24 & 58.92 & 2.61 \\
\hline PE 32:2 $16: 1(Z 9) / 16: 1(Z 9)$ & 3.78 & 59.46 & 3.91 \\
\hline PE 33:1 16:0/17:1(Z11) & 5.46 & 59.08 & 3.21 \\
\hline PG 34:2 $16: 1(Z 9) / 18: 1(Z 11)$ & 1.26 & 59.93 & 6.26 \\
\hline PE 34:2 $16: 1(Z 9) / 18: 1(Z 11)$ & 9.24 & 60.54 & 2.68 \\
\hline PE 34:1 16:0/18:1(Z11) & 20.59 & 59.24 & 2.40 \\
\hline PG 34:1 16:0/18:1(Z11) & 5.46 & 61.02 & 3.58 \\
\hline PE 32:1 16:0/16:1(Z9) & 34.03 & 59.61 & 2.01 \\
\hline PG 32:1 16:0/16:1(Z9) & 2.94 & 60.86 & 4.07 \\
\hline PG 32:0 16:0/16:0 & 1.26 & 58.45 & 5.74 \\
\hline PG 36:2 18:1(Z11)/18:1(Z11) & 2.10 & 60.70 & 4.50
\end{tabular}

Table S3. Composition and properties of the Phage PRD1 membrane (see also Figure 4).

\begin{tabular}{|c|c|c|c|}
\hline lipid nomenclature & concentration (\%) & area/lipid & std error \\
\hline PG 34:2 16:1(Z9)/18:1(Z11) & 3.87 & 66.53 & 4.30 \\
\hline PE 34:1 16:0/18:1(Z11) & 8.17 & 63.03 & 2.53 \\
\hline PE 32:2 16:1(Z9)/16:1(Z9) & 4.30 & 63.71 & 3.63 \\
\hline PE 36:2 18:1(Z11)/18:1(Z11) & 4.73 & 65.54 & 3.37 \\
\hline PE 34:2 16:1(Z9)/18:1(Z11) & 6.88 & 63.03 & 2.96 \\
\hline PG 32:1 16:0/16:1(Z9) & 13.33 & 65.39 & 2.42 \\
\hline PG 34:1 16:0/18:1(Z11) & 15.70 & 64.89 & 1.70 \\
\hline PE 30:1 14:0/16:1(Z9) & 3.44 & 62.46 & 4.51 \\
\hline PE 32:1 16:0/16:1(Z9) & 22.37 & 62.36 & 1.73 \\
\hline PG 30:0 14:0/16:0 & 4.30 & 62.17 & 3.69 \\
\hline PG 36:2 18:1(Z11)/18:1(Z11) & 5.59 & 63.27 & 3.74 \\
\hline PG 33:1 16:0/17:1(Z11) & 3.44 & 62.84 & 4.07 \\
\hline PE 33:1 16:0/17:1(Z11) & 3.87 & 59.16 & 2.88 \\
\hline
\end{tabular}


Table S4. Composition and properties of Chlorella spp. membrane (see also Figure 4).

\begin{tabular}{llcc} 
lipid nomenclature & concentration (\%) & area/lipid & std error \\
\hline PA 36:6 18:3(Z9,12,15)/18:3(Z9,12,15) & 2.14 & 68.61 & 4.23 \\
\hline PG 34:2 16:0/18:2(Z9,12) & 3.21 & 70.15 & 4.14 \\
\hline PI 36:4 18:2(Z9,12)/18:2(Z9,12) & 2.14 & 75.53 & 5.80 \\
\hline PS 36:2 18:0/18:2(Z9,12) & 2.14 & 61.36 & 3.96 \\
\hline PS 36:2 18:1(Z9)/18:1(Z9) & 1.07 & 65.23 & 6.81 \\
\hline PG 34:1 16:0/18:1(Z9) & 3.21 & 73.96 & 2.65 \\
\hline PE 36:4 18:1(Z9)/18:3(Z9,12,15) & 2.14 & 68.38 & 3.56 \\
\hline PC 32:1 16:0/16:1(Z7) & 5.35 & 72.10 & 3.76 \\
\hline PC 34:3 16:1(Z9)/18:2(Z9.12) & 0.53 & 77.94 & 14.06 \\
\hline PC 34:2 16:0/18:2(Z9.12) & 3.74 & 71.79 & 6.68 \\
\hline PC 34:1 16:0/18:1(Z9) & 7.49 & 68.00 & 1.57 \\
\hline PS 36:1 18:0/18:1(Z9) & 2.14 & 68.01 & 5.04 \\
\hline PA 36:5 18:2(Z9,12)/18:3(Z9,12,15) & 2.14 & 70.08 & 6.12 \\
\hline PE 36:5 18:2(Z9,12)/18:3(Z9,12,15) & 2.14 & 65.26 & 4.35 \\
\hline PI 36:5 18:2(Z9,12)/18:3(Z9,12,15) & 1.60 & 69.86 & 1.06 \\
\hline PE 36:4 18:1(Z9)/18:3(Z6,9,12) & 2.14 & 71.00 & 7.35 \\
\hline PG 36:3 18:1(Z9)/18:2(Z9,12) & 2.14 & 65.59 & 2.27 \\
\hline PI 34:2 16:0/18:2(Z9,12) & 1.07 & 66.81 & 5.86 \\
\hline PG 36:4 18:2(Z9,12)/18:2(Z9,12) & 3.21 & 70.71 & 4.13 \\
\hline PA 36:4 18:2(Z9,12)/18:2(Z9,12) & 1.07 & 69.06 & 8.88 \\
\hline PC 36:6 18:3(Z9,12,15)/18:3(Z9.12.15) & 8.56 & 76.24 & 3.05 \\
\hline PC 36:3 18:1(Z9)/18:2(Z9.12) & 5.35 & 69.59 & 3.28 \\
\hline PC 36:1 18:0/18:1(Z9) & 2.14 & 69.83 & 3.10 \\
\hline PC 36:5 18:2(Z9,12)/18:3(Z9.12.15) & 3.21 & 76.52 & 3.17 \\
\hline PE 36:5 18:2(Z9,12)/18:3(Z6,9,12) & 2.14 & 67.87 & 6.58 \\
\hline PC 36:2 18:1(Z9)/18:1(Z9) & 4.28 & 68.80 & 4.29 \\
\hline PE 36:2 18:1(Z9)/18:1(Z9) & 3.21 & 74.43 & 3.62 \\
\hline PE 36:3 18:1(Z9)/18:2(Z9,12) & 4.28 & 70.53 & 4.21 \\
\hline PC 36:4 18:2(Z9.12)/18:2(Z9.12) & 3.74 & 72.23 & 3.50 \\
\hline PC 36:4 18:1(Z9)/18:3(Z9.12,15) & 5.35 & 69.45 & 5.96 \\
\hline PC 32:1 16:0/16:1(Z9) & 5.88 & 71.00 & 2.67 \\
\hline PC 34:3 16:1(Z7)/18:2(Z9.12) & & 65.79 & 9.60 \\
\hline
\end{tabular}




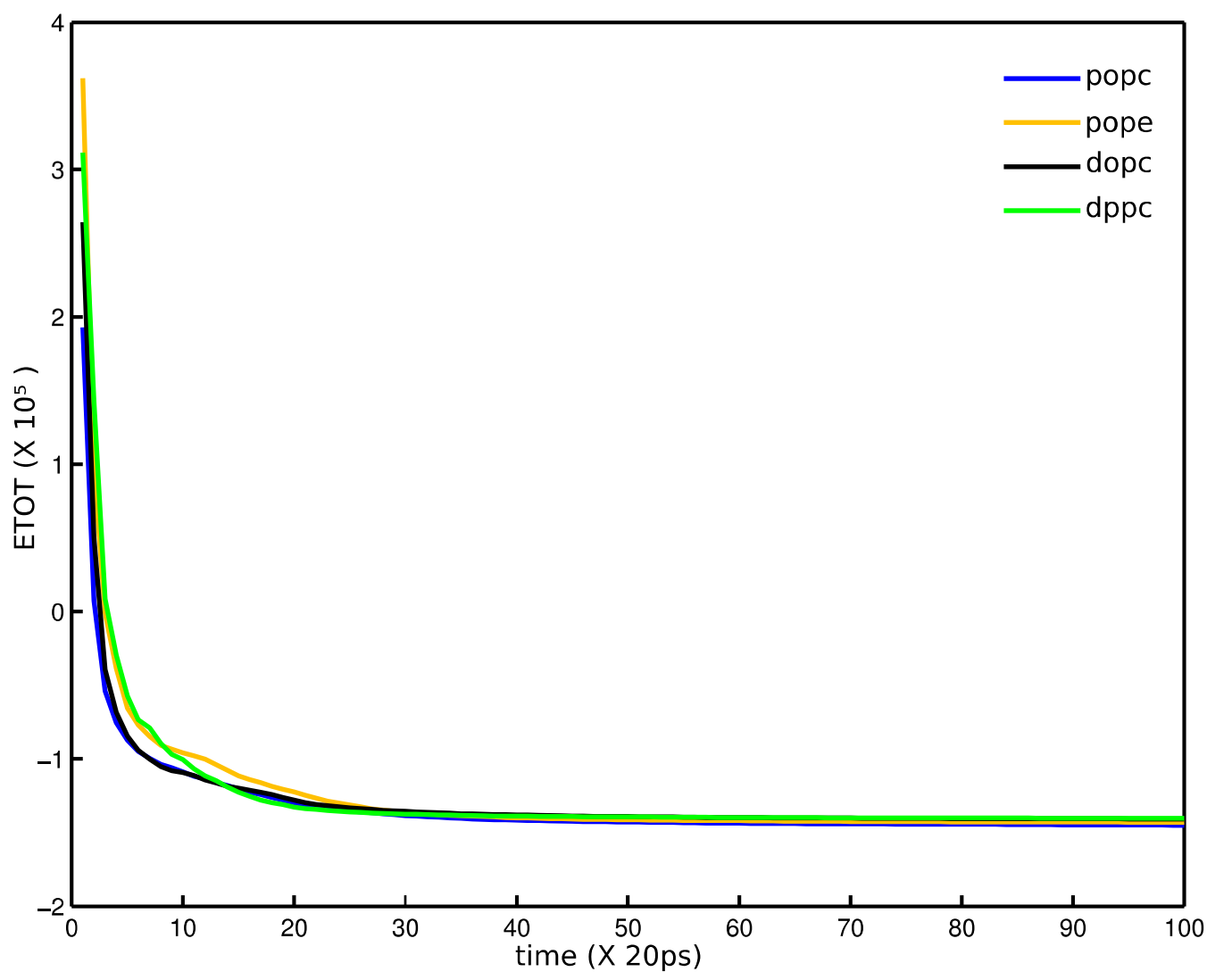

Figure S1. Evolution of total energies (ETOT) of the pure membrane (POPC,POPE,DOPC and DPPC) systems through the initial molecular dynamics minimization. 
Figure S2. Chemical structure of lipids composing the phage PRD1 membrane system.
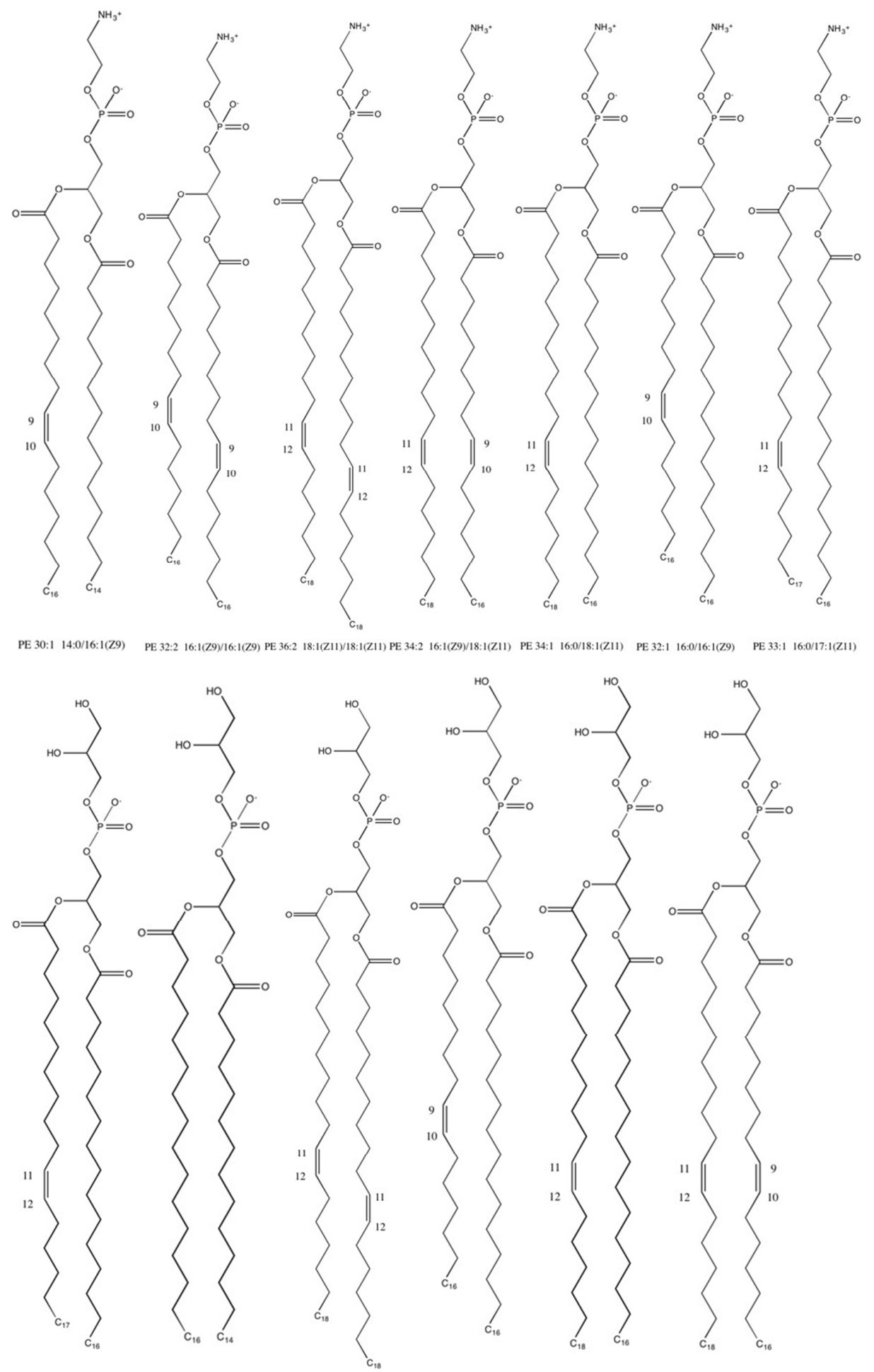

PG 33:1 16:0/17:1(Z11)

PG 30:0 14:0/16:0

PG 36:2 18:1(Z11)/18:1(Z11) PG 32:1 16:0/16:1(Z9)

PG 34:1 16:0/18:1(Z11) PG 34:2 16:1(Z9)/18:1(Z11) 
Figure S3. Chemical structure of lipids composing the Salmonella enterica membrane.

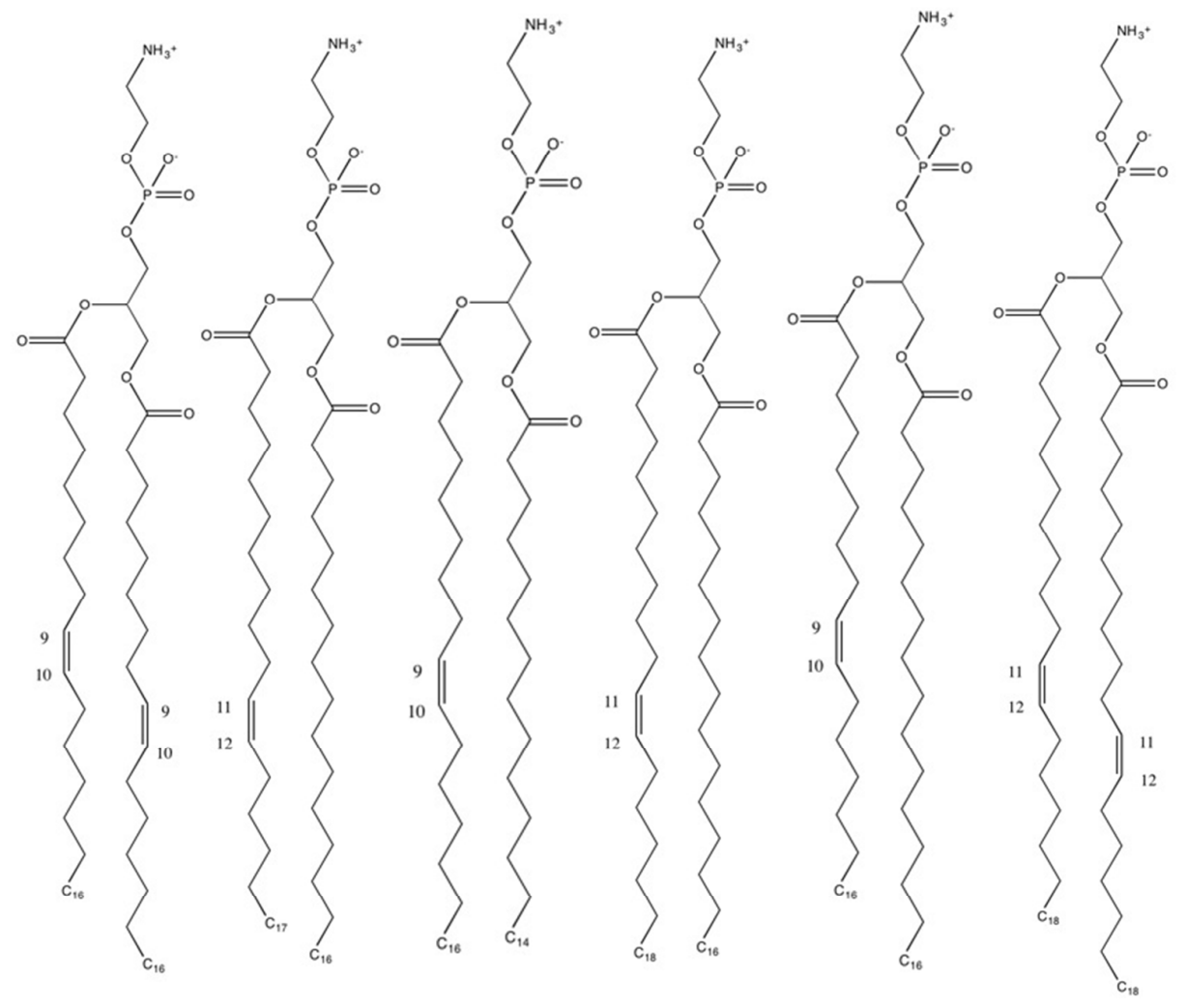

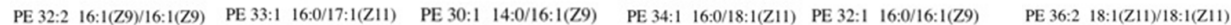




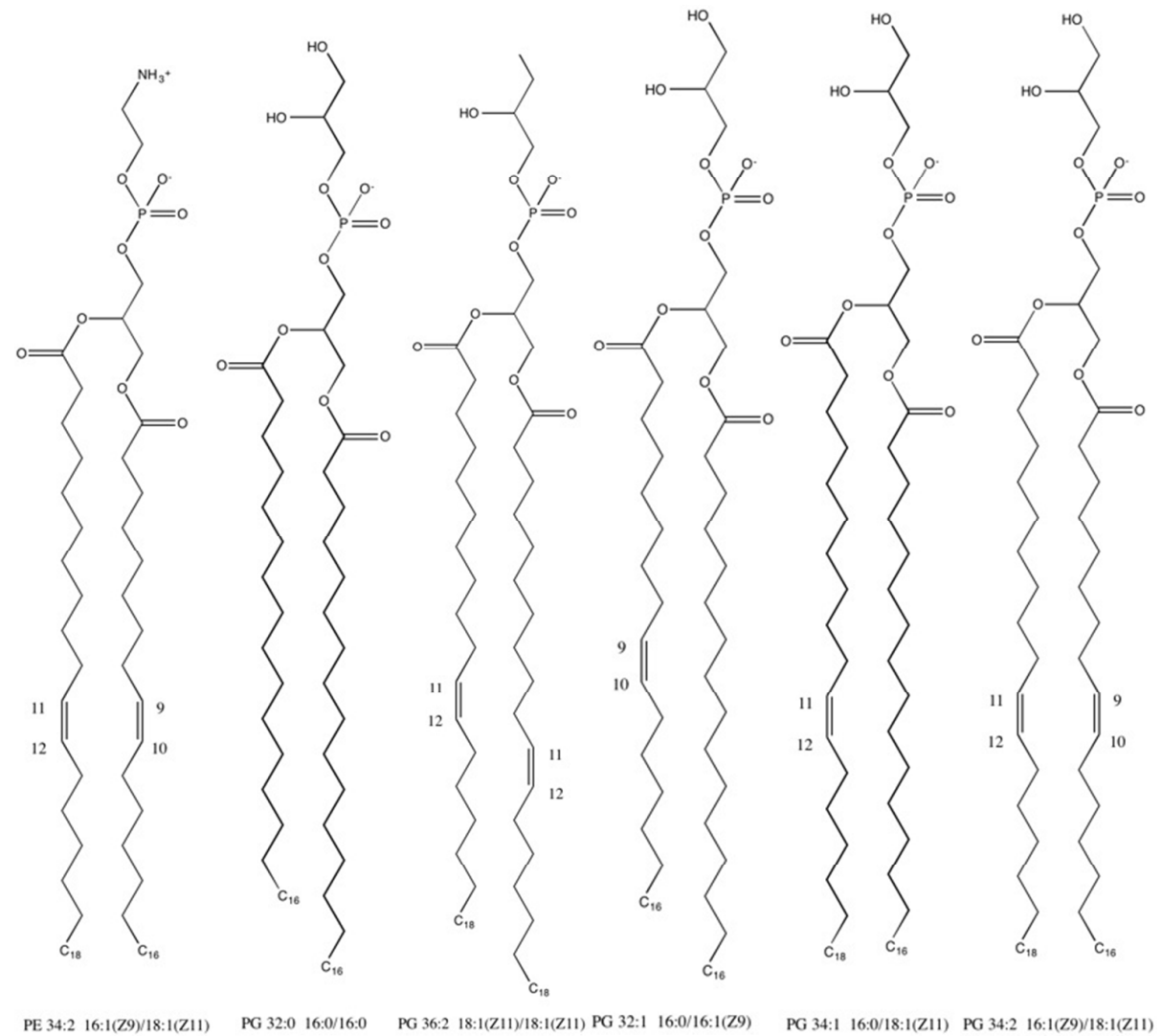

Figure S4. Chemical structure of lipids composing the Chlorella spp. membrane.

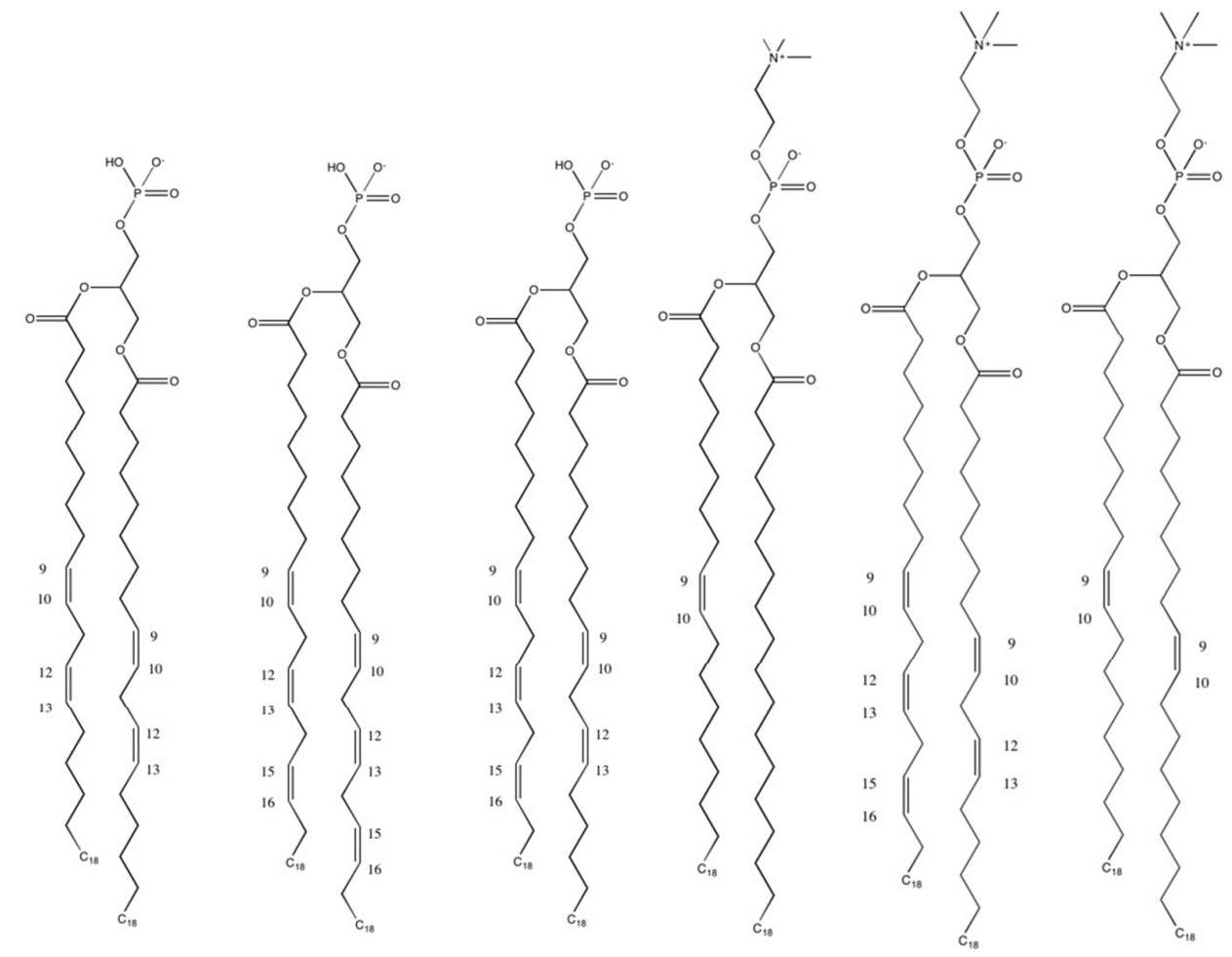

PA 36:4 18:2(Z9,12)/18:2(Z9,12) PA 36:6 18:3(Z9,12,15)/18:3(Z9,12,15) PA 36:5 18:2(Z9,12)/18:3(Z9,12.15) PC 36:1 18:0/18:1(Z9) PC 36:5 18:2(Z9,12)/18:3(Z9.12.15) PC 36:2 18:1(Z9)/18:1(Z9) 


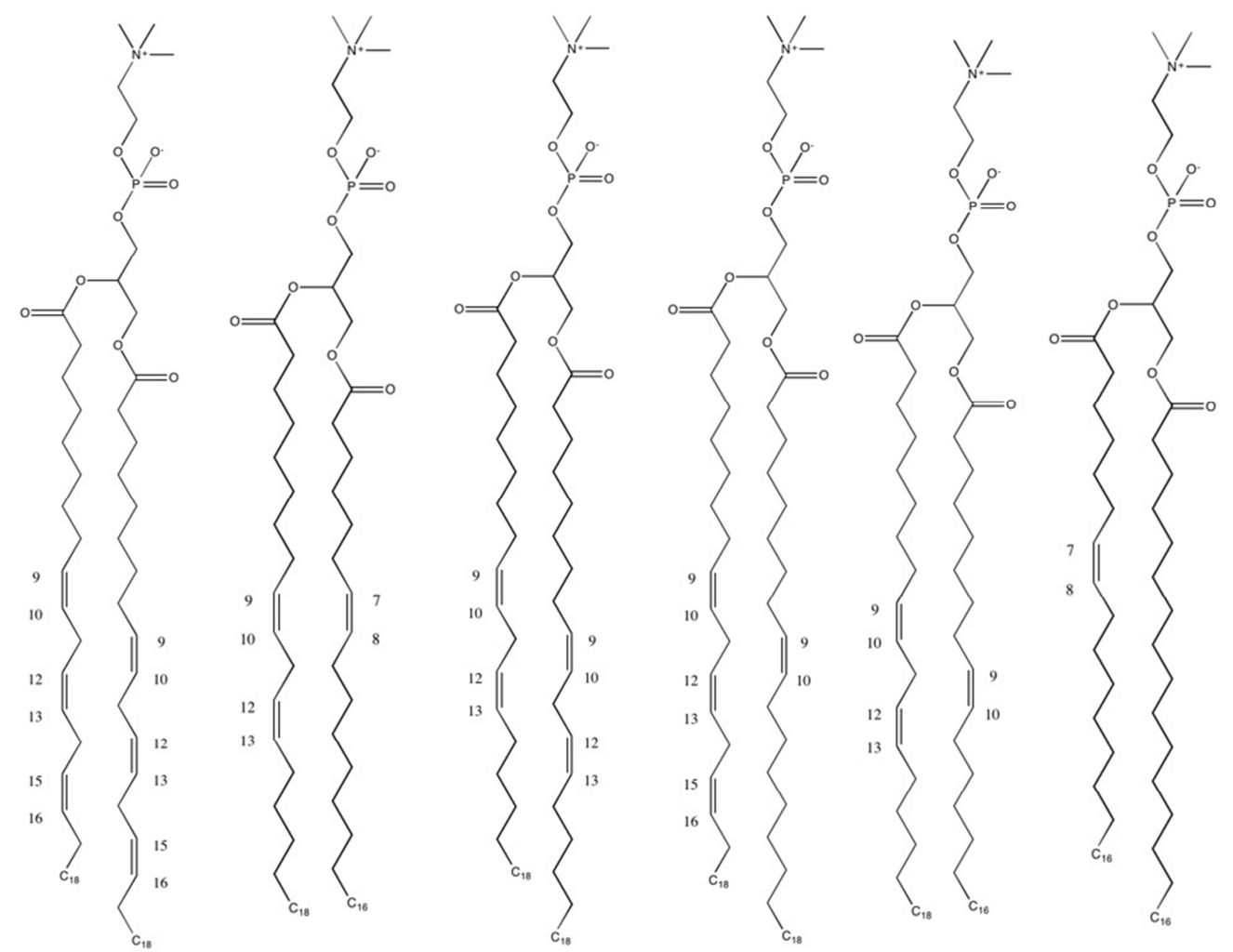

PC 36:6 18:3(Z9,12,15)/18:3(Z9.12.15) PC 34:3 16:1(Z7)/18:2(Z9.12) PC 36:4 18:2(Z9.12)/18:2(Z9.12) PC 36:4 18:1(Z9)/18:3(Z9.12.15) PC 34:3 16:1(Z9)/18:2(Z9.12) PC 32:1 16:0/16:1(Z7)

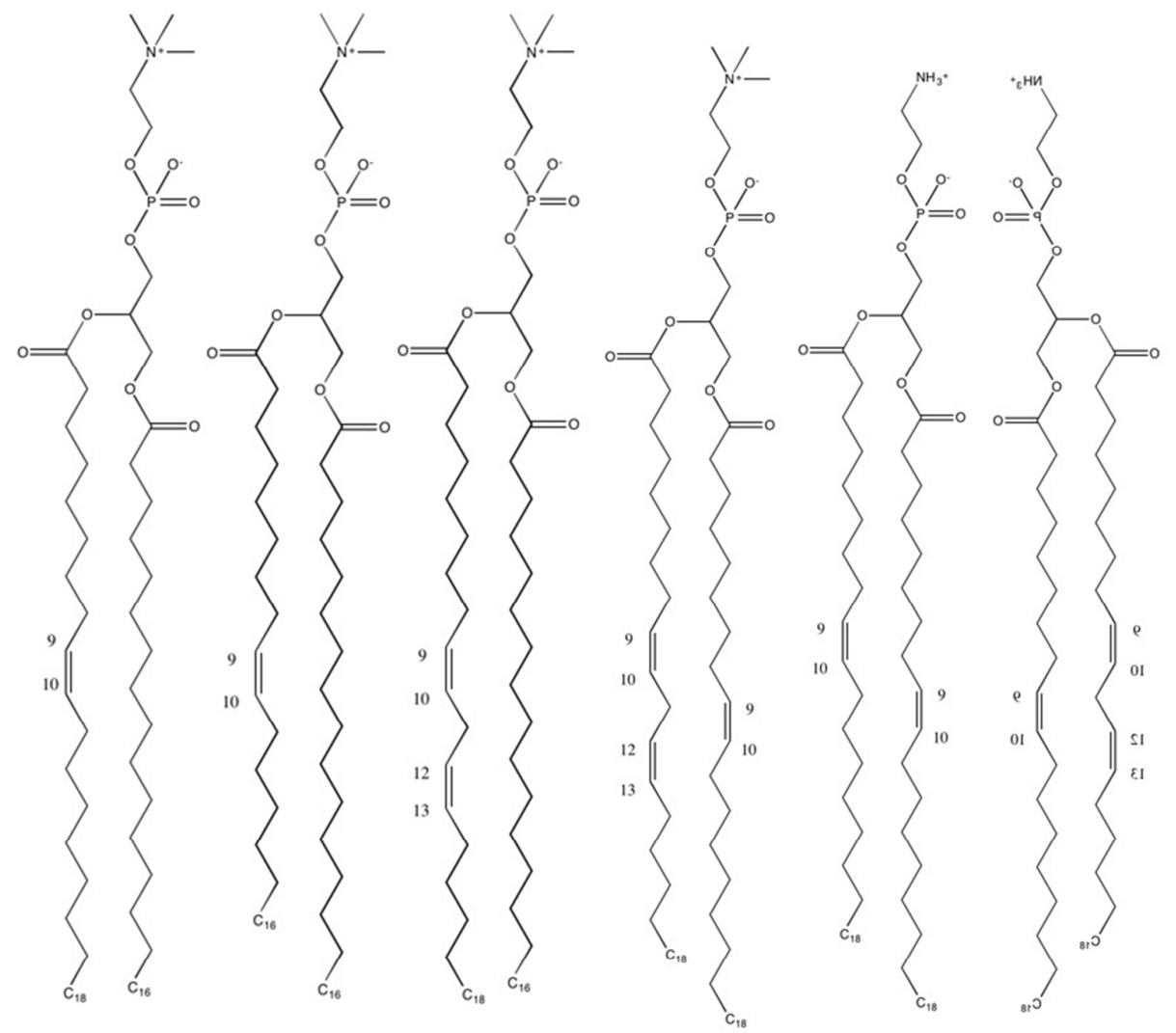

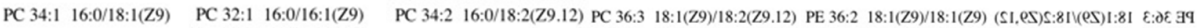




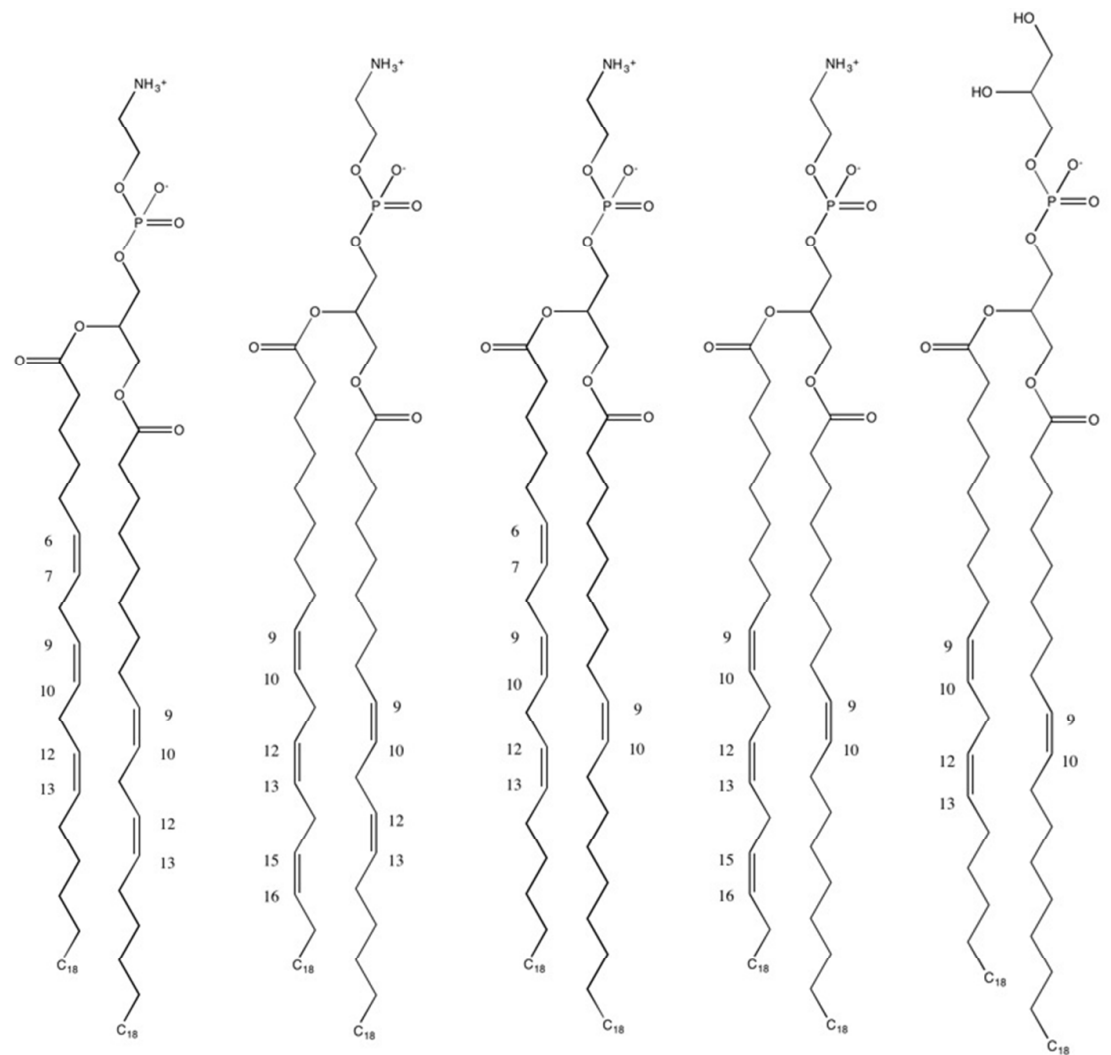

PE 36:5 18:2(Z9.12)/18:3(Z6.9.12) PE 36:5 18:2(Z9,12)/18:3(Z9,12,15) PE 36:4 18:1(Z9)/18:3(Z6.9,12) PE 36:4 18:1(Z9)/18:3(Z9.12.15) PG 36:3 18:1(Z9)/18:2(Z9,12)

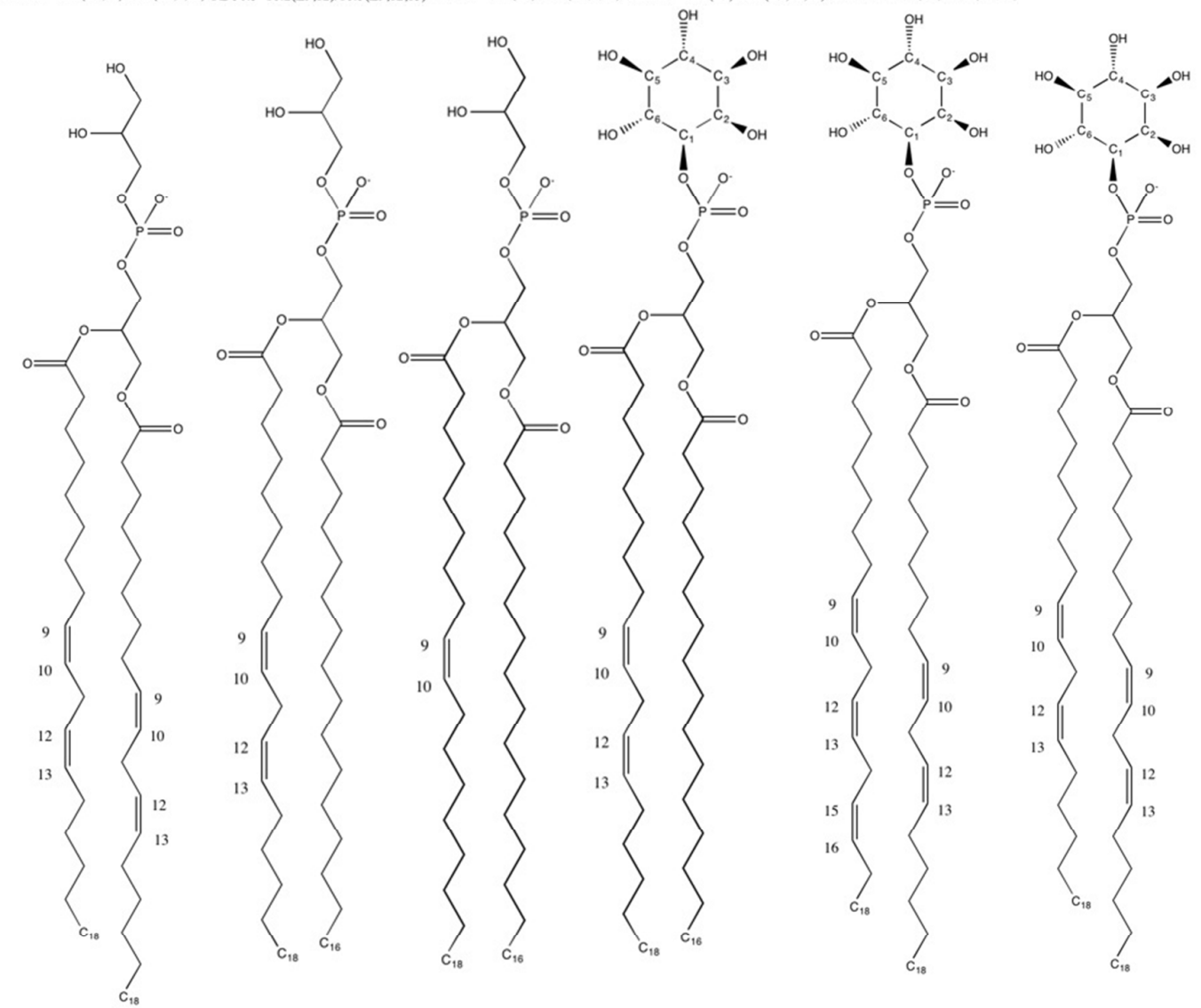




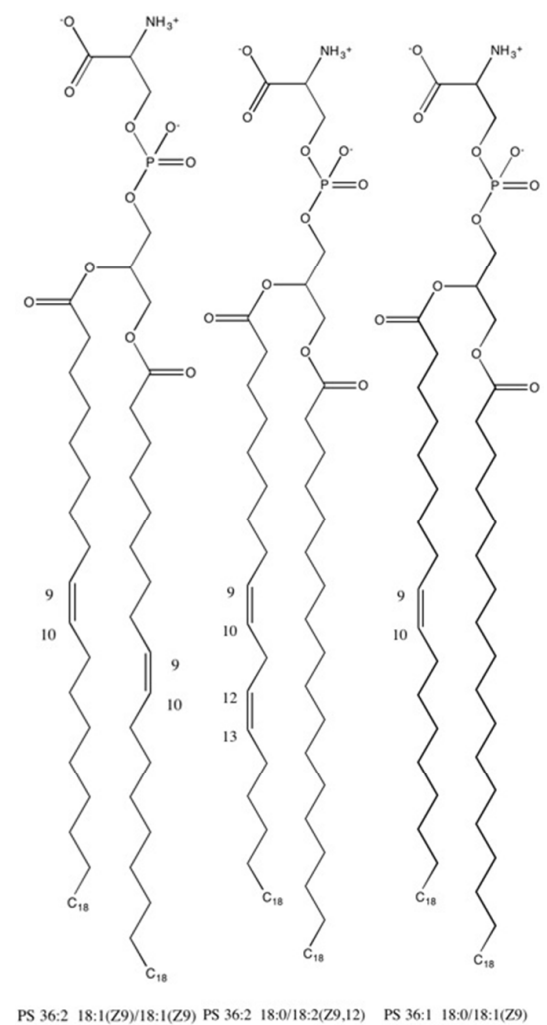

